\title{
Primary acinic cell carcinoma of the breast is associated with a poor outcome: A case report and literature review
}

\author{
LUDIVINE SARSIAT $^{1}$, GEORGE WATKINSON ${ }^{2}$, ARRAN TURNBULL ${ }^{2}$, \\ ANNA DIANA $^{3}$ and OLGA OIKONOMIDOU ${ }^{2}$ \\ ${ }^{1}$ Department of Pharmaceutical Sciences, University of Bordeaux, 33076 Bordeaux, France; \\ ${ }^{2}$ Cancer Research UK Edinburgh Centre, Institute of Genetics and Cancer, \\ University of Edinburgh, Edinburgh EH4 2XR, UK; \\ ${ }^{3}$ Department of Precision Medicine, Campania University 'Luigi Vanvitelli', I-80131 Naples, Italy
}

Received May 13, 2020; Accepted March 31, 2021

DOI: $10.3892 / \mathrm{mco} .2021 .2476$

\begin{abstract}
Primary acinic cell carcinoma (AcCC) is a rare histological type of malignant breast cancer. AcCC was first identified as an entity in 1996, and since then 51 cases have been reported in the literature. The first early case reports and reviews suggested a relatively favourable prognosis for patients with AcCC; however, reports of AcCC recurrent disease have been more recently described in a subset of patients with high-grade disease. The present case report describes an unusual case of estrogen receptor-negative AcCC of the breast in a 59-year-old woman who did not respond to neoadjuvant chemotherapy (NACT), despite imaging revealing a large reduction in tumour volume. Furthermore, 14 months after NACT completion, the patient presented with disease progression comprising peritoneal involvement and linitis plastica. The patient started on first-line chemotherapy with carboplatin and paclitaxel combination, achieving a notable and prolonged response. After 2.5 years and while still on carboplatin and paclitaxel, the patient developed leptomeningeal carcinomatosis disease (LD) and died 6 weeks after LD presentation. The present report is the third case of AcCC in which cancer-associated death was registered. As studies on large series are lacking, further investigations are required to identify predictors of poor outcome. Notably, the prolonged response achieved to first-line chemotherapy suggested that platinum and taxane compounds may offer a potential therapeutic benefit for patients with AcCC. Moreover, the present case report highlights the importance of careful interpretation of follow-up imaging, as an apparent positive response to treatment may not always be a true representation of disease.
\end{abstract}

Correspondence to: Dr Olga Oikonomidou, Cancer Research UK Edinburgh Centre, Institute of Genetics and Cancer, University of Edinburgh, Crewe Road South, Edinburgh EH4 2XR, UK

E-mail: olga.oikonomidou@ed.ac.uk

Key words: acinic cell carcinoma, breast cancer, salivary gland-like tumour

\section{Introduction}

Primary acinic cell carcinoma (AcCC) of the breast was first identified as an entity by Roncaroli et al (1) in 1996 and since then 51 cases have been reported in the literature (1-30).

It is a very rare subtype of the salivary gland-like tumour group that occurs in breast tissue. This group comprises three sub-categories: Tumours displaying pure myoepithelial cell differentiation, such as myoepitheliomas; tumours with mixed epithelial and myoepithelial cell differentiation, such as pleomorphic adenoma, adenomyoepithelioma and adenoid cystic carcinoma; and tumours showing pure epithelial cell differentiation, such as acinic cell carcinoma, oncocytic carcinoma, mucoepidermoid carcinoma and polymorphous adenocarcinoma (31).

Breast AcCC shares many classical features with salivary gland counterpart, with frequent expression of S-100, lysozyme, salivary-type amylase, and alpha-1-antichimotrypsin positive (A1-ACT) and periodic acid-Schiff (PAS) positivity in addition (32). Specific risk factors for AcCC of breast are still unknown. However, it is primarily observed in women after the age of 40 years (the mean age of presentation is around 55 years). Furthermore, based on its similarity with the salivary gland counterpart, previous radiation exposure as well as familial history of breast cancer could represent an important risk factor for developing this rare type of breast malignancy.

The first early case reports and reviews suggested a relatively favourable prognosis for patients with AcCC, even though this variant is often of the triple negative breast cancer (TNBC) subtype on immunohistochemistry. However, reports of AcCC recurrence cases have been more recently published $(10,13,24,25)$. Based on available data, the prognosis seems to be largely driven by the presence of poorly differentiated components in these tumours. Furthermore, in the majority of cases, patients affected by AcCC received chemotherapy and radiotherapy as adjuvant treatment, as the optimal therapeutic strategy has not yet been established for this rare variant of breast cancer.

Here, we report an unusual case of high grade, Estrogen Receptor (ER) negative AcCC associated with poor response 
to anthracycline and taxane based neo-adjuvant chemotherapy (NACT), a rapid disease progression within a short time from NACT completion and a prolonged progression free survival (PFS) on combination regimen of Carboplatin and Paclitaxel.

\section{Case report}

A 59-year-old woman presented to the Western General Hospital, Edinburgh, UK, having noticed a lump in her right breast. Urgent mammography identified a 30x22×30 mm ill-defined solid lesion that corresponded anatomically with the mass clinically palpated. A further mass inferior to this measuring $15 \times 8 \mathrm{~mm}$ was also identified. Multiple simple cysts were seen in both breasts and a mammography performed two years ago as part of a screening programme, showed that these opacities were long-standing benign changes.

Tissue analysis of a breast core biopsy revealed an invasive carcinoma grade 3 exhibiting areas of necrosis focally associated with atypical acinar structures containing brightly eosinophilic secretions reminiscent of microglandular adenosis (MGA) at the peripheries of the tumour.

Immunohistochemically, the specimen including collections of glandular differentiation areas showed a lack of myoepithelial markers such as CK14 and p63. A stain for ER was focal and weakly positive (Allred score 2) while negative for Progesterone receptor (PR) and Human epidermal growth factor receptor 2 (Her2). No carcinoma in-situ or lymphovascular invasion was observed.

The patient received six cycles of NACT with 3 cycles of FEC-100 (Epirubicin $100 \mathrm{mg} / \mathrm{m}^{2}$ with 5-Fluorouracil $500 \mathrm{mg} / \mathrm{m}^{2}$ and Cyclophosphamide $500 \mathrm{mg} / \mathrm{m}^{2}$ every 21 days) followed by 3 cycles of Docetaxel $100 \mathrm{mg} / \mathrm{m}^{2}$. At the end of the 6 cycles a repeated mammogram and ultrasound of the breast confirmed a large reduction in volume of the main lesion with no significant residual mass seen on scans. No nodes were identified in the right axilla. This was considered a good radiological response to treatment.

Three weeks later, the patient proceeded to a right breast wide local excision (WLE) and sentinel node biopsy (SNB). Pathology report confirmed the previous diagnosis of invasive carcinoma showing glandular cell-like features. Sentinel lymph nodes were negative $(0 / 2)$ for invasive carcinoma with no evidence of fibrosis suggesting no previous infiltration from cancer (TNM stage ypT2, ypN0 (sn)). Macroscopic analysis of the 40x50x40 mm sample identified vaguely cream-coloured tissue with yellowish flecks within posterior tissue.

Microscopically, a circumscribed tumour focus composed of solid and cohesive nests of cells displaying a more spindle cell morphology in places as well as some glandular differentiation with foci of mucin production was observed at the medial margin. However, elsewhere, the tumour displayed a more dispersed and infiltrative appearance and included irregular cords of cells and some single cell infiltration and areas with coalescence and proliferation of solid cell nests showing variable eosinophilic granular cytoplasm. The areas with a microglandular appearance presented densely eosinophilic luminal secretions and formed some coalescing sheets, configuring the typical features of MGA. No conventional ductal carcinoma in situ was identified. Infiltrative elements with a more solid and nested appearance were seen within $<1 \mathrm{~mm}$ from the tumour margins. There was a variable nuclear pleomorphism up to nuclear score 3 and patchy high mitotic rate giving a mitotic score of 3. Overall, the acinar score was of 2. These appearances were in keeping with features of grade 3 disease (Fig. 1).

Despite some variation in tumour cellularity and focal fibrosis compared to the initial biopsy analysis, there was no obvious vascular fibrosis associated with any significant decrease in cellularity present to indicate any definite response to NACT.

Further analysis using immunohistochemistry showed that myoepithelial cell markers (CK14, CK5/6, p63, SMA, Calponin) were negative. The tumour showed immunopositivity for S100, epithelial membrane antigen (EMA), amylase and gross cystic disease fluid protein 15 (GCDFP-15), as well as focal positivity to CD68. Neuroendocrine markers, CD56, chromogranin and synaptophysin were all negative. Tumour cells were negative for ER and HER2 but positive for PR with variable staining. Overall estimated PR score was 4 but was up to 6 in some foci. All margins were found to be positive of residual invasive carcinoma. Re-excision of all margins revealed residual tumour elements, leading to an increase in tumour volume. No therapeutic response to NACT was observed. The patient subsequently underwent right mastectomy and the tissue analysis reported further residual disease extending beyond all original margins with similar appearances to the tumour in previous specimens. The whole size of the lesion was estimated to be at least $71 \mathrm{~mm}$ in the largest diameter.

After surgery, the patient proceeded with chest wall adjuvant radiotherapy as per standard protocol. No further treatment was offered at that time and she received annual surgical follow up and contralateral annual mammography.

Unfortunately, 14 months later, the patient presented with upper abdominal distention and pain. Gastroscopy revealed Linitis Plastica. Peritoneal and gastric biopsies were taken and confirmed infiltration by a carcinoma with similar features to the primary breast cancer. Immunohistochemistry also revealed an identical profile to that seen in the first breast core biopsy with neoplastic cells showing immunopositivity for CK7, GATA3, EMA, amylase, E-Cadherin, GCDFP and S-100 and immunonegativity for ER, CK20 and CDX2. PR status was regarded as negative since the cells displayed patchy aberrant cytoplasmic staining. The patient had full staging with a CT scan of chest, abdomen and pelvis (CAP) and a bone scan. CT CAP showed extensive peritoneal and serosal disease with biliary tree dilatation, pleural effusions and ascites. The bone scan did not reveal any bone metastases.

In view of disease progression, performance status (PS) deterioration and highly deranged liver enzymes (LFTs), the patient was started initially on weekly Paclitaxel as first line chemotherapy. After 2 weeks, all parameters including PS and LFTs improved and weekly Carboplatin AUC2 was added to Paclitaxel. Overall, the patient achieved an impressive response for 30 months with several breaks in chemotherapy over the time, not due to toxicities but for treatment holiday purposes. This is interesting given that the patient had no response to neo-adjuvant Docetaxel. 


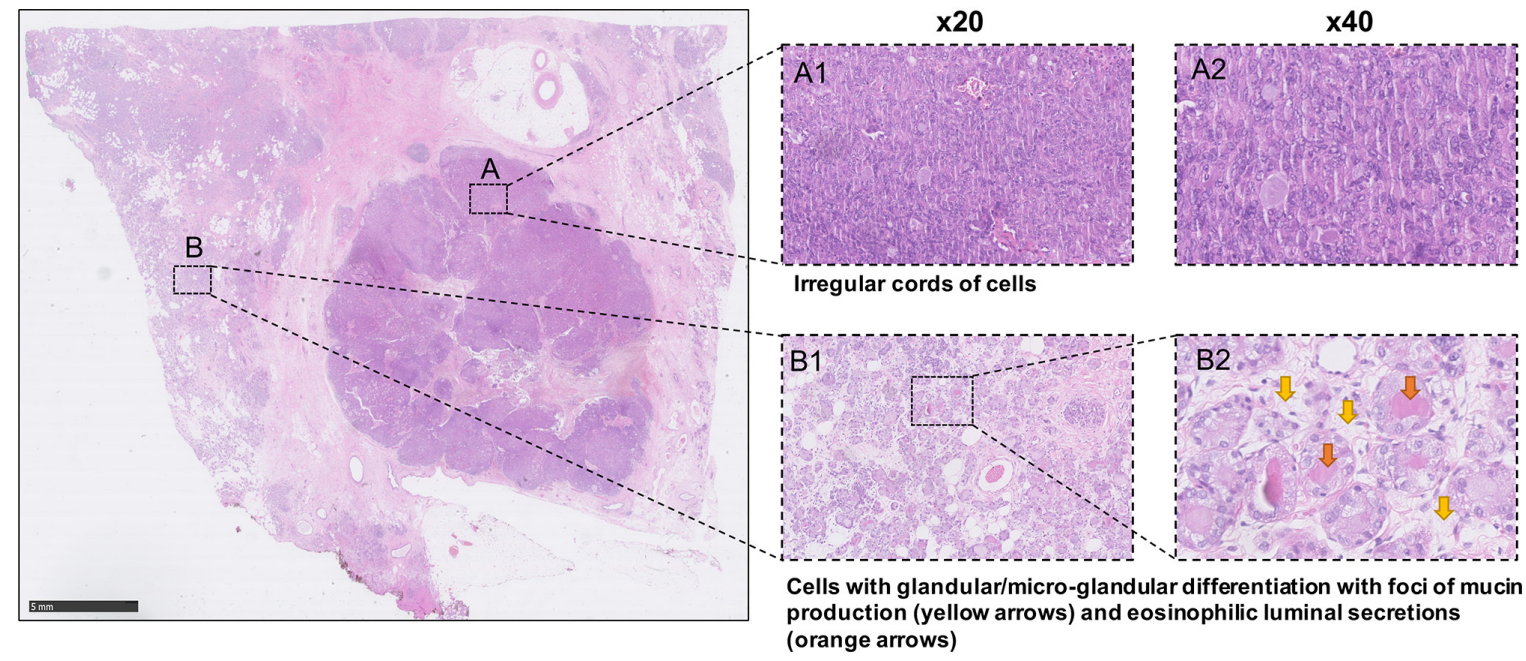

Figure 1. Histological aspect of invasive acinic cell carcinoma. The tumour (gastric biopsy collected for diagnostic purposes) displayed irregular cords of cells, as well as solid and cohesive nests of cells with glandular differentiation (scale bar, $5 \mathrm{~mm}$ ). (A) Irregular cords of cells (magnifications, $\mathrm{x} 20$ and $\mathrm{x} 40$ ). (B) Cells with glandular/micro-glandular differentiation with foci of mucin production (yellow arrows) and eosinophilic luminal secretions (orange arrows) (magnifications, $\mathrm{x} 20$ and $\mathrm{x} 40$ ).

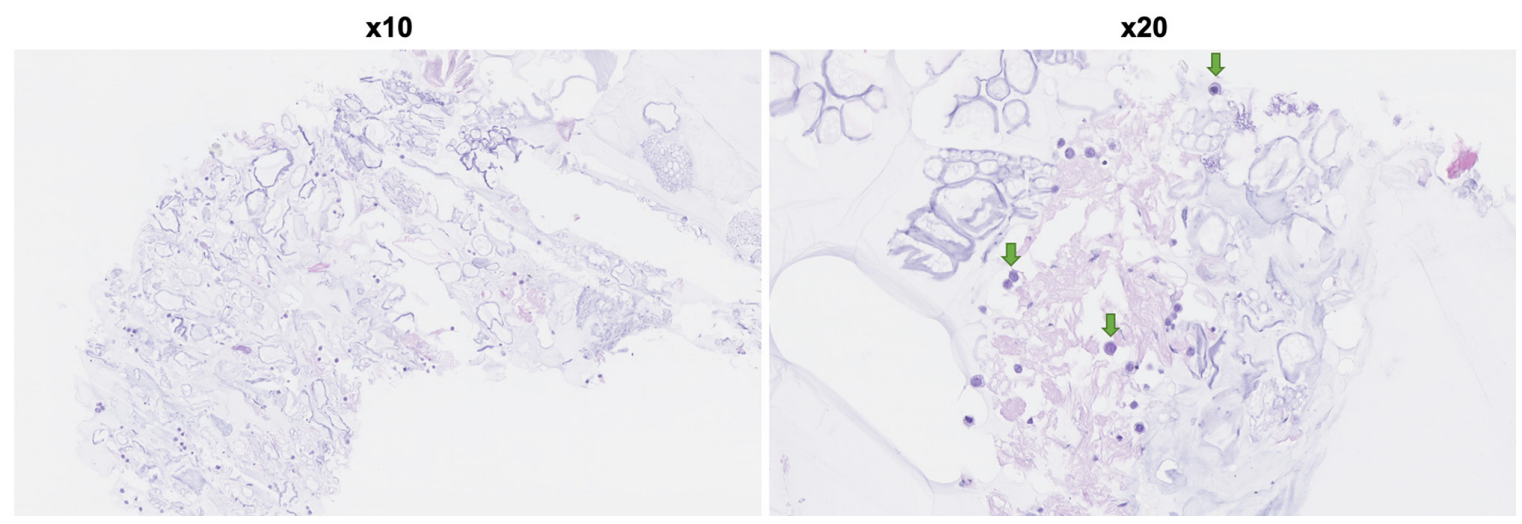

Figure 2. Cerebral spinal fluid analysis. Cytospin and cell block preparations showed pleomorphic cells as both adhesive and individual groups (green arrows), which immunoreacted with cytokeratin 7 and GATA3, but were negative for cytokeratin 20. Magnifications, x10 and x20.

Two and a half years after the diagnosis of metastatic AcCC, and while on Paclitaxel/Carboplatin and having an excellent response with regards to her visceral disease, the patient presented with excruciating headaches and a CT scan of the head was performed. The CT showed no evidence of intracranial metastases. Subsequently a head MRI was requested and a lumbar puncture was performed. The MRI head showed radiological evidence of leptomeningeal carcinomatosis (Fig. 2). The lumbar puncture showed malignant cells in the cerebrospinal fluid. Cytospin and cell block preparations revealed pleomorphic cells as both adhesive and individual groups, which immunoreacted with cytokeratin 7 and GATA3, but were negative for cytokeratin 20. The patient's PS was very poor and decision against further treatment was made. At that time, she was transferred to a hospice setting where she died 6 weeks later.

\section{Discussion}

Breast and salivary glands are both composed of tubuloacinar exocrine glands, and as such they share similar cytology and morphology to normal healthy tissue. Therefore, in the event of disease they may exhibit similar characteristics.

We have presented a case report of breast cancer displaying characteristic salivary gland-like features. The association of a microglandular and a solid growth pattern, the presence of bright eosinophilic cytoplasmic granularity and the immuno-profile are most in keeping with acinic cell-like carcinoma, according to the criteria outlined by Roncaroli (1) and Damiani (3). The main immunohistochemical features reported in the literature on breast AcCC in a subset of cases with detailed immunohistochemical profile description $(n=36)$ are summarised in Table I. The presence of coarse bright eosinophilic cytoplasmic granules in breast epithelium is rare. It has mostly been described in MGA lesions and in AcCC carcinoma (25). Both present a similar morphology, positivity for S-100 and absence of myoeptithelial markers. However, they can be differentiated by immunohistochemistry since AcCC shows positivity for EMA, lysozyme, alpha amylase and A1-ACT, whereas MGA does not. Additionally, MGA typically features a basal lamina that is absent in AcCC. MGA is known to be a benign breast lesion but can 
Table I. Summary of the immunohistochemical features reported in the literature on breast AcCC.

\begin{tabular}{lcc}
\hline $\begin{array}{l}\text { Immunohistochemical features of breast } \\
\text { AcCC }\end{array}$ & $\begin{array}{c}\text { Positivity, \% (number of cases/total cases) } \\
\text { (present case included) }\end{array}$ & $\begin{array}{c}\text { Feature present }(+) / \text { Absent }(-) \text { in } \\
\text { the present case }\end{array}$ \\
\hline PAS (diastase resistant) & $100(24 / 24)$ & + \\
S-100 & $87(27 / 31)$ & + \\
Lysozyme & $96(23 / 24)$ & + \\
Epithelial membrane antigen & $100(21 / 21)$ & + \\
Amylase & $95(19 / 20)$ & NR \\
$\alpha-1-$ Antitrypsin & $54(6 / 11)$ & NR \\
$\alpha-1-$ Antichymotrypsin & $78(11 / 14)$ & NR \\
Cytokeratin 7 & $100(9 / 9)$ & - \\
Neuroendocrine markers (synaptophysin) & $15(2 / 13)$ & + \\
Gross cystic disease fluid protein 15 & $50(9 / 18)$ & - \\
Estrogen receptor & $13(4 / 31)$ & + \\
Progesterone receptor & $22(7 / 31)$ & NR \\
Androgen receptor & $11(1 / 9)$ & - \\
HER2 & $0(0 / 25)$ & - \\
Triple-negative carcinoma & $72(18 / 25)$ & + \\
\hline
\end{tabular}

AcCC, acinic cell carcinoma; PAS, periodic acid-Schiff; NR, not reported.

be associated with breast carcinoma up to $27 \%(33,34)$. Its relationship with AcCC remains unclear, but some authors have postulated that MGA may be a precursor lesion of AcCC (35). In the present case, it is difficult to be certain whether some of the acinar areas may represent pre-existing MGA.

The present case showed immunopositivity for PAS, S-100, lysozyme and EMA, as in almost all AcCC described in literature, but also for GCDFP-15, a marker of apocrine differentiation expressed in one-half of the cases. Moreover, the current case displayed positivity of PR that was observed in only $20 \%$ of reported cases (Table I). To date, as summarized in Table II, nine cases of hormonal receptor positive AcCC have been described. The mean age at diagnosis was 53 years with a single case involving a male patient (2). Of these, only one patient experienced local recurrence of disease and, subsequently, lymph node metastases after radical surgery, adjuvant radiotherapy and systemic therapies (25). Similarly to our case, tumour exhibited parallel cytological spectrum where the solid and nested infiltrative areas had all high-grade features of triple-negative breast carcinomas whereas the well-differentiated acinar-like components displayed lower mitotic rate and nuclear pleomorphism score (25).

The majority of AcCC breast cancer cases reported in the literature are triple negative tumours (36). However, recently, some authors described rare cases of AcCC showing positivity for both estrogen and progesterone receptors (Table II).

In the present case, the biopsy sample showed a very weak immunoreactivity for ER (Allred score 2) while the tissue from WLE specimen following NACT displayed no signal for this receptor. On the contrary, PR result was negative at the analysis of core biopsy but positive with variable staining (Allred score 4) when assessed on the WLE specimen. This could be explained by the greater accuracy of the pathology assessment on a WLE specimen rather than the biopsy. A change of the receptor status could also be due to the chemotherapy, and perhaps this, rather than accuracy of sample analysis, could underlie the difference in the expression of HRs before and after NACT. Interestingly, biopsies of the recurrent disease exhibited similar features to the diagnostic biopsy rather than the surgical specimen.

Although the majority of AcCC are of the triple negative subtype, early case reports and the first few reviews of breast AcCC cases reported it to be a tumour with a mostly favourable outcome. However, more recent reviews have identified that this is not always the case, and suggest that there is a sub-group of patients with higher-grade tumours who have a very poor outcome $(6,10)$.

As reported in Table III, of the 52 cases available in the literature (including the present case), nine patients experienced complications following adjuvant treatment, such as local recurrence, metastases, or death. Distant metastases have been reported in five cases, and death consequent to tumour progression occurred in three patients. However, follow up period is not available for all patients and for some of them it is probably too short to detect local or distant recurrences. It is noteworthy that most of the patients have been treated with chemotherapy and radiotherapy in addition to surgery.

The current case is the third of AcCC in which cancer-related death was registered. This unfavorable outcome could be related to the presence of a grade 3 large cancer and the triple negative nature of the tumour.

To date, there is a lack of consensus in terms of the appropriate systemic treatment and the effect of chemotherapy on 


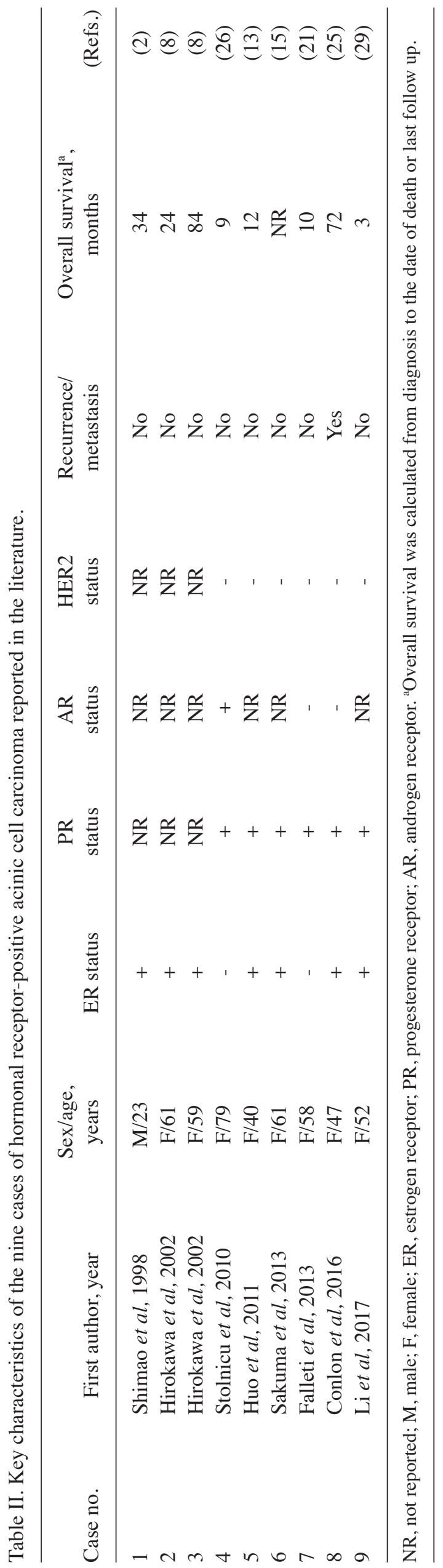

tumour cells in breast AcCC is not currently well known. There are only five reports in the literature of patients with AcCC that have received NACT $(3,6,13,19,25)$. Comparative descriptions of specimens pre- and post-NACT have been performed in 4 out of the 5 cases $(3,6,13,19,25)$. In these series, the response to NACT has not been specified, except in the case published by Winkler et al (19) in which a lack of chemotherapeutic effect was described in surgical specimen as well as at MRI imaging, after four cycles of Adriamycin followed by four cycles of Paclitaxel. As discussed above no response to anthracycline and taxane based NACT was observed in this case either.

Furthermore, immunohistochemically, the omnipresence of coarse bright eosinophilic cytoplasmic granules confirms the fact that these are features of the tumour and not a chemotherapy effect. Interestingly, a significant increase in presence of these granules was observed after chemotherapy in three of reported cases $(6,19,25)$. Conversely, in all cases where intraductal carcinoma (IDC) was diagnosed with concurrent AcCC and in which NACT was given, the solid poorly differentiated cells of the IDC, which were predominant in the pretreatment core biopsy specimen, were residual or absent after treatment. This leads the authors to postulate a strong chemosensitivity of solid poorly differentiated carcinoma cells, and a possible chemoresistance of the microglandular component of these tumours.

In our case, the presence of eosinophilic granules was also identified both in the breast biopsy and the mastectomy specimen without any cellularity decrease seen after therapy. Moreover, the apparent decrease in tumour volume observed at follow-up mammogram following NACT could be due to clearance of the infiltrative solid nests of cells, against which chemotherapy may be more effective at cell kill than against microglandular areas.

Finally, another point of interest is the prolonged response (30 months) that the patient achieved by receiving Carboplatin in combination with Paclitaxel as first line chemotherapy, despite the marked chemoresistance of the tumour that has been previously observed in neo-adjuvant setting.

Since ER and PR status was regarded as negative at the time of peritoneal biopsy, the reason of this impressive and unexpected response could lie in the well-known sensitivity of the TNBC subtype to the Platinum salts and Taxanes (37).

In conclusion, primary $\mathrm{AcCC}$ is a rare type of breast carcinoma and in the majority of cases is classified as triple negative subtype. There is a growing body of evidence that AcCC is not always associated with a good prognosis, as believed until now, and further investigations are required to identify predictors of poor outcome. Here, we have presented the case of a patient with ER negative AcCC with no response to conventional NACT. Despite being triple negative, AcCC are not considered as very chemosensitive tumours. However, the combination of Carboplatin and Paclitaxel might offer a therapeutic benefit and significantly prolong the progression free survival as shown in this case. Our case also highlights the importance of careful interpretation of follow-up imaging, as an apparent positive response to treatment may not always be a true representation of disease. 


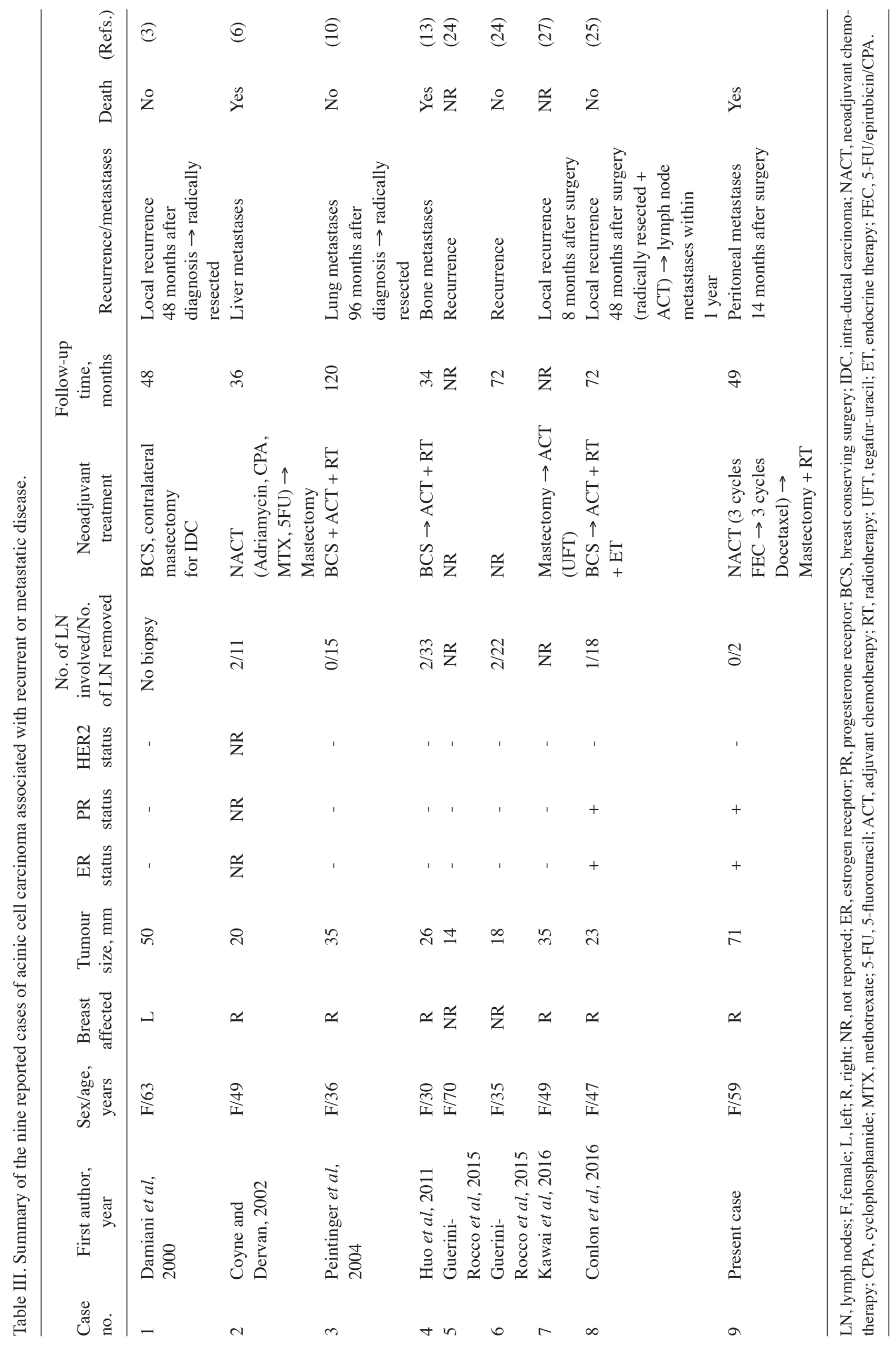




\section{Acknowledgements}

Not applicable.

\section{Funding}

No funding was received.

\section{Availability of data and materials}

Data sharing is not applicable to this article, as no datasets were generated or analyzed during the current study.

\section{Authors' contributions}

LS, GW, AT, AD and OO conducted the literature review and wrote/edited the final manuscript. OO provided all clinical information relevant to this case. OO and AT confirm the authenticity of all the raw data. All authors have read and approved the final manuscript.

\section{Ethics approval and consent to participate}

Not applicable.

\section{Patient consent for publication}

Written informed consent was obtained from the patient for publication of this case report before her death. Additionally, the patient donated tissue and blood samples for translational research.

\section{Competing interests}

The authors declare that they have no competing interests.

\section{References}

1. Roncaroli F, Lamovec J, Zidar A and Eusebi V: Acinic cell-like carcinoma of the breast. Virchows Arch 429: 69-74, 1996.

2. Shimao K, Haga S, Shimizu T, Imamura H, Watanabe O, Kinoshita J, Nagumo H, Utada Y, Okabe T, Kajiwara T, et al: Acinic cell adenocarcinoma arising in the breast of a male: A clinicopathological, immunohistological and ultrastructural study. Breast Cancer 5: 77-81, 1998.

3. Damiani S, Pasquinelli G, Lamovec J, Peterse JL and Eusebi V: Acinic cell carcinoma of the breast: An immunohistochemical and ultrastructural study. Virchows Arch 437: 74-81, 2000.

4. Schmitt FC, Riberio CA, Alvarenga S and Lopes JM: Primary acinic cell-like carcinoma of the breast-a variant with good prognosis? Histopathology 36: 286-289, 2000.

5. Chang ED, Lee EJ, Lee AW, Kim JS and Kang CS: Primary acinic cell carcinoma of the breast: A case report with an immunohistochemical and ultrastructural studies. J Breast Cancer 14 160-164, 2011.

6. Coyne JD and Dervan PA: Primary acinic cell carcinoma of the breast. J Clin Pathol 55: 545-547, 2002.

7. Elster EA, Markusic J, Ball R, Soballe P, Henry M, Louie A and Clare S: Primary acinic cell carcinoma of the breast. Am Surg 68: 993-995, 2002.

8. Hirokawa M, Sugihara K, Sai T, Monobe Y, Kudo H, Sano N and Sano T: Secretory carcinoma of the breast: A tumour analogous to salivary gland acinic cell carcinoma? Histopathology 40: 223-229, 2002.

9. Kahn R, Holtveg H, Nissen F and Holck S: Are acinic cell carcinoma and microglandular carcinoma of the breast related lesions? Histopathology 42: 195-196, 2003.
10. Peintinger F, Leibl S, Reitsamer R and Moinfar F: Primary acinic cell carcinoma of the breast: A case report with long-term follow-up and review of the literature. Histopathology 45: 645-648, 2004.

11. Kinkor Z and Skálová A: Acinic cell-like differentiation in invasive ductal carcinoma and in ductal hyperplasia of the breast-report of two cases. Cesk Patol 41: 29-33, 2005 (In Czech).

12. Tanahashi C, Yabuki S, Akamine N, Yatabe Y and Ichihara S: Pure acinic cell carcinoma of the breast in an 80-year-old Japanese woman. Pathol Int 57: 43-46, 2007.

13. Huo L, Bell D, Qiu H, Sahin A, Wu Y and Sneige N: Paneth cell-like eosinophilic cytoplasmic granules in breast carcinoma. Ann Diagn Pathol 15: 84-92, 2011.

14. Choh CT, Komar V and Courtney SP: Primary acinic cell carcinoma of the breast: A rare lesion with good prognosis. Breast J 18: 610-611, 2012.

15. Sakuma T, Mimura A, Tanigawa $\mathrm{N}$ and Takamizu R: Fine needle aspiration cytology of acinic cell carcinoma of the breast. Cytopathology 24: 403-405, 2013.

16. Zhao Y, Li W, Lang R, Yang Y, Gao X, Zheng Y, Zhang C, Fu X and Fu L: Primary acinic cell carcinoma of the breast: A case report and review of the literature. Int J Surg Pathol 22: 177-181, 2014.

17. Shingu K, Ito T, Kaneko G and Itoh N: Primary acinic cell carcinoma of the breast: A clinicopathological and immunohistochemical study. Case Rep Oncol Med 2013: 372947, 2013.

18. Osako T, Takeuchi K, Horii R, Iwase T and Akiyama F: Secretory carcinoma of the breast and its histopathological mimics: Value of markers for differential diagnosis. Histopathology 63: 509-519, 2013.

19. Winkler N, Morrell G and Factor R: Invasive carcinoma with acinic cell-like features of the breast. Breast J 19: 334-335, 2013.

20. Ripamonti CB, Colombo M, Mondini P, Siranoush M, Peissel B, Bernard L, Radice P and Carcangiu ML: First description of an acinic cell carcinoma of the breast in a BRCA1 mutation carrier: A case report. BMC Cancer 13: 46, 2013.

21. Falleti J, Coletti G, Rispoli E, Scarabeo F, Cervasio M, Tornillo L, Pettinato G and Insabato L: Acinic cell carcinoma of the breast arising in microglandular adenosis. Case Rep Pathol 2013: 736048, 2013.

22. Limite G, Di Micco R, Esposito E, Sollazzo V, Cervotti M, Pettinato G, Varone V, Benassai G, Monda A, Luglio G, et al: The first case of acinic cell carcinoma of the breast within a fibroadenoma: Case report. Int J Surg 12 (Suppl 1): S232-S235, 2014.

23. Piscuoglio S, Hodi Z, Katabi N, Guerini-Rocco E, Macedo GS, $\mathrm{Ng} \mathrm{CK}$, Edelweiss M, De Mattos-Arruda L, Wen HY, Rakha EA, et al: Are acinic cell carcinomas of the breast and salivary glands distinct diseases? Histopathology 67: 529-537, 2015.

24. Guerini-Rocco E, Hodi Z, Piscuogloio S, Ng CK, Rakha EA, Schultheis AM, Marchiò C, da Cruz Paula A, De Filippo MR, Martelotto LG, et al: The repertoire of somatic genetic alterations of acinic cell carcinomas of the breast: An exploratory, hypothesis-generating study. J Pathol 237: 166-178, 2015.

25. Conlon N, Sadri N, Corben AD and Tan LK: Acinic cell carcinoma of breast: Morphologic and immunohistochemical review of a rare breast cancer subtype. Hum Pathol 51: 16-24, 2016.

26. Stolnicu S, Dohan M, Preda O, Goez E, Cabrero IA and Nogales FF: Primary acinic cell carcinoma of the breast associated with an intraductal acinic cell component. Patología 48: 204-207, 2010.

27. Kawai H, Sugimoto R, Iga N, Ikeda H, Yoshida R, Waki N, Ishizaki M, Nishi H and Yamashita K: A case of primary acinic cell carcinoma (ACC) of the breast. Gan To Kagaku Ryoho 43: 2019-2021, 2016 (In Japanese).

28. Sherwell-Cabello S, Maffuz-Aziz A, Rios-Luna NP, Bautista-Piña V and Rodríguez-Cuevas S: Salivary gland-like breast carcinomas: An infrequent disease. Pathol Res Pract 212: 1034-1038, 2016.

29. Li H, Wang F, Shen P and Zhou F: Pure acinic cell carcinoma of the breast: A case report and literature review. Medicine (Baltimore) 96: e8866, 2017.

30. Sen R, Bhutani N, Kamboj J and Dahiya S: Primary acinic cell carcinoma of the breast: A case report with a clinicopathological and immunohistochemical study of a rare breast cancer subtype. Ann Med Surg (Lond) 35: 137-140, 2018.

31. Tavassoli FA and Eusebi V (eds): Tumors of the Mammary Gland. American Registry of Pathology, Washington, DC, pp212-260, 2009. 
32. Foschini MP, Morandi L, Asioli S, Giove G, Corradini AG and Eusebi V: The morphological spectrum of salivary gland type tumours of the breast. Pathology 49: 215-227, 2017.

33. Salarieh A and Sneige N: Breast carcinoma arising in microglandular adenosis: A review of the literature. Arch Pathol Lab Med 131: 1397-1399, 2007.

34. Khalifeh IM, Albarracin C, Diaz LK, Symmans FW, Edgerton ME, Hwang RF and Sneige N: Clinical, histopathologic, and immunohistochemical features of microglandular adenosis and transition into in situ and invasive carcinoma. Am J Surg Pathol 32: 544-552, 2008

35. Shui R and Yang W: Invasive breast carcinoma arising in microglandular adenosis: A case report and review of the literature. Breast J 15: 653-656, 2009.
36. Lakhani SR, Ellis IO, Schnitt SJ, Tan PH and van de Vijver MJ (eds): WHO classification of tumors of the breast. IARC, Lyon, 2012.

37. Curigliano G: Addition of platinum salts to neoadjuvant chemotherapy in triple-negative breast cancer: A new standard of care? Lancet Oncol 19: 434-436, 2018.

cc) (i) (9) This work is licensed under a Creative Commons EY No No Attribution-NonCommercial-NoDerivatives 4.0 International (CC BY-NC-ND 4.0) License. 